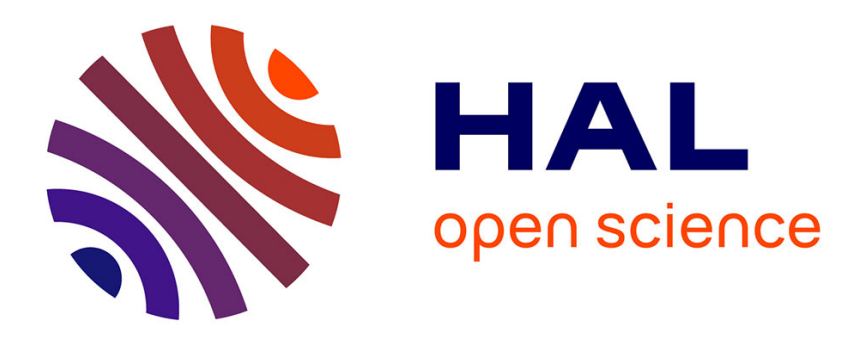

\title{
Evaluation of a maintenance strategy by the analysis of the rate of repair
}

François Pérès, Daniel Noyes

\section{To cite this version:}

François Pérès, Daniel Noyes. Evaluation of a maintenance strategy by the analysis of the rate of repair. Quality and Reliability Engineering International, 2003, vol. 19 ( $\left.\mathrm{n}^{\circ} 2\right)$, pp. 129-148. 10.1002/qre.515. hal-01296527

\section{HAL Id: hal-01296527 \\ https://hal.science/hal-01296527}

Submitted on 1 Apr 2016

HAL is a multi-disciplinary open access archive for the deposit and dissemination of scientific research documents, whether they are published or not. The documents may come from teaching and research institutions in France or abroad, or from public or private research centers.
L'archive ouverte pluridisciplinaire HAL, est destinée au dépôt et à la diffusion de documents scientifiques de niveau recherche, publiés ou non, émanant des établissements d'enseignement et de recherche français ou étrangers, des laboratoires publics ou privés. 


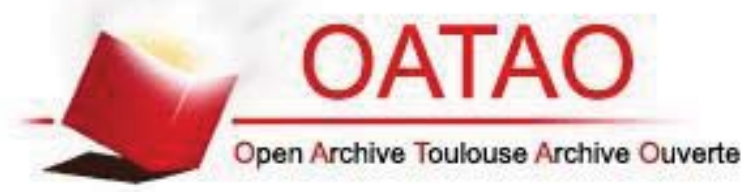

\section{Open Archive TOULOUSE Archive Ouverte (OATAO)}

OATAO is an open access repository that collects the work of Toulouse researchers and makes it freely available over the web where possible.

This is an author-deposited version published in : http://oatao.univ-toulouse.fr/ Eprints ID : 10311

To link to this article : DOI : 10.1002/qre.515

URL : http://dx.doi.org/10.1002/qre.515

To cite this version : Pérès, François and Noyes, Daniel Evaluation of a maintenance strategy by the analysis of the rate of repair. (2003) Quality and Reliability Engineering International, vol.19 (n॰2). pp.129-148. ISSN 0748-8017

Any correspondance concerning this service should be sent to the repository administrator: staff-oatao@listes-diff.inp-toulouse.fr 


\title{
Evaluation of a Maintenance Strategy by the Analysis of the Rate of Repair
}

\author{
François Pérès $1, *, \dagger$ and Daniel Noyes ${ }^{2}$ \\ ${ }^{1}$ Laboratoire Productique Logistique, Ecole Centrale Paris, Grande Voie des Vignes, 92295 Châtenay-Malabry Cedex, \\ France \\ ${ }^{2}$ Laboratoire Génie de Production, Ecole Nationale d'Ingénieurs de Tarbes, 47, avenue d'Azereix B.P. 1629 \\ 65016 Tarbes Cedex, France
}

The evaluation of maintenance strategies is a problem which has been widely tackled. However, it remains a topic worthy of consideration since the economic stakes peculiar to this activity are huge. The work presented in this paper deals with this problem under the point of view of data feedback. A statistical analysis is made from the examination of the forms of the failure rate distribution. The laws resulting from the implementation of a given maintenance strategy inform us about the relevance of the choices representative of this strategy. The construction of these laws from a raw time of repair is a global approach which tends to hide the induced phenomenon. A decomposition of the repair process into phases allows us to better identify the influence of the strategy parameters. The analysis of each elementary phase distribution is interesting since it gives information about the performance of the implemented maintenance strategy.

KEY WORDS: maintenance strategy; rate of repair; statistical analysis; performance evaluation

\section{INTRODUCTION}

$\mathrm{I}$

$\mathrm{n}$ this paper, we present a methodology for the evaluation of maintenance strategies by taking into consideration the effect of certain variables on the dynamic of maintenance, on its structure and its context of evolution. Our approach considers the return to an operational state as a point of entry into the evaluation of a strategy. The approach is factual. It is based on the treatment of data collected from the history of the behaviour of equipment on which the strategy to be evaluated can be applied. The paper is divided into three parts. Literature in the field of maintenance is explored in the first part, from the aspect of strategies used and some particular characteristics of the forms of failure and repair. The second part is a synthesis of the techniques of the collection and processing of factual data, at the foundation of evaluation of the strategy. The method of evaluation is presented in the last part. It is a structure with three stages. First, we point out and describe the aggregated presence of elementary phases in the corrective and preventive processes. We then propose a series of rules of decomposition of a time of repair for the characterization of the durations of each elementary

*Correspondence to: François Pérès, Laboratoire Productique Logistique, Ecole Centrale Paris, Grande Voie des Vignes, 92295 ChâtenayMalabry Cedex, France.

†E-mail: Francois.peres@enit.fr 
phase when the information which allows their estimation is not, or is only partially, available. Lastly, from the knowledge of the influence of the maintenance parameters on the elementary times and of the sensitivity of the system to their variation, we propose an approach which leads to the evaluation of the corresponding maintenance strategy. The ensemble of developed concepts in this work is applied to a practical case which corresponds to a real industrial situation.

\section{MODELLING OF MAINTENANCE AND FAILURE/REPAIR PROCESSES IN THE CURRENT LITERATURE}

\subsection{Maintenance}

The strategies of maintenance are as diverse and varied as are the systems of production to which they are applied. Beyond their differences, the objective of each of these strategies is to maintain the system of production in a working condition as long as possible or to restore it as quickly as possible in the case of failure.

To begin with, a distinction is to be made between the works which treat the interventions of perfect maintenance $^{1,2}$ (making the system as good as new) and imperfect maintenance ${ }^{1,3}$ (repairing the system to a less deteriorated state, but without completely eliminating the damage). Certain authors ${ }^{4,5}$ imagine the two scenarios with the maintenance being sometimes perfect with the probability of $p$, sometimes imperfect with the probability of $1-p$. Other authors 6,7 , speak of minimal maintenance to describe an intervention which brings the system into a less deteriorated state, but without specifying the level of residual deterioration. The term corrective maintenance is applied to the maintenance strategy which restores the system to a pre-failure state ${ }^{3}$. The term 'systematic preventive maintenance' is employed to describe preventive interventions implemented on fixed dates and with constant intervals. Conditional preventive maintenance is characterized by maintenance interventions carried out after the detection of signals emitted by the system revealing present or imminent dysfunction ${ }^{8}$.

Some authors attempt to respond to the most basic questions which are asked by the maintainers: should we intervene in the system or not? If so, is it preferable to repair or to replace the failed component ${ }^{9,10}$ ? Other works, less direct in a choice of a single strategy, consider more complex combinations.

The concept of major maintenance has been developed by Sim and Endreneyi ${ }^{6}$, who defined this maintenance as a perfect intervention after $x$ minimal interventions. They show, in particular, that this type of maintenance is useless if the intervals between minimal maintenance interventions are already optimal. Similarly, other works introduce the notion of opportunistic maintenance ${ }^{11-13}$. This particular type of maintenance permits one, on a multi-component system, to take advantage of a failure occurrence of one of the components which is then repaired or replaced and then carry out preventive inspections of the neighbouring components at the same time. Vineyard and Meredith ${ }^{14}$ envisaged several strategies of combinations of opportunistic and corrective maintenance. Hopp and $\mathrm{Wu}^{15}$ made another division. They distinguished between deterministic maintenance and stochastic maintenance. The difference appears at the level of the result of the intervention, which is considered as known in the first case and random in the second.

Gupta and Chand ${ }^{16}$ put forward the idea of a strategy of temporary replacement of failed components by components which have already been used, but which had been previously replaced after having reached a threshold limit (age, for example). This work introduced the concept of palliative maintenance. Finally, Pellegrin ${ }^{4}$ combined systematic and conditional maintenance. Conditional maintenance, in fact, proves to be very efficient for certain components which emit precursor signs of failure, but is inadequate for others. The choice to combine the two types of preventive maintenance, then, could appear to be logical. Paté-Cornell et $a .^{7}$ defined the level of the maintenance action to be performed as depending on the interpretation of the signals emitted by the system.

\subsection{Forms of failure}

The 'failure' is the manifestation of an 'error' which follows a 'fault'. In the literature, these faults are generally considered as being of systemic origin, resulting from the phenomena of wear, fatigue, infant mortality or can 
be purely random. Other types of faults could appear in the form of, for example, human faults ${ }^{17-19}$ or design faults ${ }^{20,21}$.

The failure of multi-component systems could be characterized by the progressive failure of a certain number of its entities or by a common failure mode on the ensemble of the $n$ components of the system ${ }^{22,23}$. In the first case, the system could be declared as failed at the occurrence of the first local failure (serial configuration) ${ }^{16}$ or at the failure of $k$ out of $n$ entities (presence of hot or cold redundance) ${ }^{24}$.

Two major classes of failures are highlighted in the literature: cataleptic failures and progressive failures. The difference lies in the rapidity in which the system falls into a state of failure. This crossing over into the failure state may either be immediate in the case of 'binary' systems (good or bad) ${ }^{25}$, or it could be in steps of progressive levels, the last level obviously being the state of failure. In this second case, the deterioration could be either discrete ${ }^{25}$ or continuous ${ }^{26}$. Discrete deterioration could be interpreted as the quantity of damage to the system following shocks, this quantity increasing by a fixed, variable or random amount on certain dates $^{27}$. Kapur and Bhalla ${ }^{28}$ make the hypothesis that the moments of occurrence of the shocks are limited, their timescale also being discrete. The number of states corresponding to the respective levels of deterioration is generally ignored, but certain works consider it as a fixed number. The failure comes after $k$ shocks or passage of the system into $k$ levels of deterioration ${ }^{6,29}$.

In this way, the number of levels increases progressively according to papers until reaching, in the case of an infinite number of shocks on a fixed horizon, the modelling of continuous deterioration. Continuous deterioration corresponds to a process of progressive and regular degradation comparable to a wear phenomenon or of fatigue ${ }^{30}$. The system is declared failed after crossing a threshold. In certain papers ${ }^{27,29,31}$ the deterioration is considered to be discrete and continuous at the same time, the failure modes resulting from the utilization of the system being superimposed on the wear phenomenon.

Preventive maintenance is only of interest to the extent in which it can be applied to a system which has a failure rate that is not constant. However, the Poissonian hypothesis, because of the simplicity of its utilization, is widely used for the modelling of the failure process ${ }^{32,33}$. Numerous works use other more realistic laws representing different failure rates. For example, an increasing rate is considered but the law is not clearly defined $^{34-36}$. Weibull's law, reputed for accurately interpreting the failure rate increasing period but which is difficult to manipulate, is only used in certain works ${ }^{37-40}$. The Erlang law also appears ${ }^{4}$. Lastly, certain works consider non-homogenous Poisson laws ${ }^{41,42}$, log-normal distributions ${ }^{40}$, the law of extreme values ${ }^{38}$ or arbitrary rates $^{43,44}$ to characterize the phenomenon of failure.

In a multi-component system, the failures of each entity generally have an influence on the reliability behaviour of the other components ${ }^{22,23}$. Albin and $\mathrm{Chao}^{45}$ considered a multi-component system with dependence. The failure of one component leads to the increase of the failure rate of the neighbouring components.

Until now, we have only referred to sources of failure emanating from the system. The maintenance resources could also be at the origin of a dysfunction, in preventing the correct and complete repair of the system to a good working state. Actually, these resources may have themselves undergone failures ${ }^{46}$ or be unavailable at the time of the system failure (multi-component system with limited maintenance resources) ${ }^{47}$. In the case of conditional maintenance, we also note the possible failure of the monitoring system ${ }^{48}$. Paté-Cornell et al. ${ }^{7}$ showed that errors in the interpretation of the results of inspection (systematic maintenance) or the signals (conditional maintenance) may lead to two types of errors characterized by the $\alpha$ and $\beta$ risks ${ }^{7}$. An error of type I consists of overestimating or 'inventing' a problem (risk $\alpha$ ), while an error of type II consists of underestimating or ignoring a problem (risk $\beta$ ). Even though error I may be considerably less harmful than error II, the two are detrimental to the functioning of the system, causing the system to be stopped without reason or continuing until the failure does indeed occur.

\subsection{Forms of repair}

Repair generally follows the failure of a system ${ }^{27}$. However, it can follow an inspection or a signal occurrence ${ }^{48}$. Its activation is immediate ${ }^{7}$ or deferred ${ }^{48}$. Because of severe production constraints, the repairs may be programmed to start at fixed dates ${ }^{49}$ and be limited in time ${ }^{50}$. This type of planification implies a dissociation 
between the nature of the repair and the gravity of the failure. More naturally, a direct relation is established between the duration of the repair and the amount of damage to the system. In a paper by Paté-Cornell et al. ${ }^{7}$, the level of repair depends on the nature of the signal emitted by the system. In contrast, other authors assume a single level of repair which brings the system back to as good as new ${ }^{1,2}$.

The location of repair is not often mentioned in the literature. Waganer ${ }^{51}$ distinguishes between the level of failure authorizing an on site repair and the level of failure necessitating the movement of the failed part to a more favourable intervention zone. The number of repairers and, more generally, the amount of resources are generally determined in order to avoid the unavailability of a repair service in the occurrence of a failure. In certain papers ${ }^{52,53}$, however, the number of repairers is restricted. It can even be limited to one single person in $^{2}$.

\section{PROCESSING OF FACTUAL DATA}

The definition and optimization of the strategies of maintenance must be supported by theoretic distributions. To this end, the maintainer must first know the nature of the laws which govern the system. Two steps are necessary, namely data collection and data processing.

\subsection{Data collection}

Data collection corresponds to the capture of information relating to the characteristics of failure and repair processes and request and service. This information may be varied. Data concerning a corrective intervention may be, for example, the date, description and duration of an intervention as well as the nature, cause and gravity of the failure. These data are then exploited for the purpose of the management and organization of maintenance.

In order to illustrate the descriptive part of the model of processing the collected information, we refer to a factual database established in an industrial environment from the failures and repair of production machines. Data are related to a numeric control machine tool shop and concerns 50 pieces of machinery (lathe and milling). The information is extracted from weekly workflow schedules established by the production or maintenance operators and represent the sequences of normal function and breakdown of the machines. The reasons for the interruptions and the operations carried out on the failed machine are specified, which then allows one to draw conclusions related to failure typology. The majority of the resources of the shop function in two shifts because of the high cost of depreciation of this type of equipment and the heavy load of production. During these periods, engagement is maximum. This engagement constraint leaves a very small window period for preventive maintenance interventions which take place on an approximately annual basis.

A great amount of similarity was recognized during the processing of a list of values which concerns the 50 machines. This constant can be linked, on the one hand, to the similar characteristics and performance of the machines, but equally to the same forms of engagement in production and to the same intervention protocols in curative and preventive maintenance. For this reason, the results and comments presented in this paper refer to the study of a single list of values (that we also call the sample of reference hereafter) which correspond to the observation over a period of six years of the functioning of a unit composed of four similar machining centres.

The results reported in Tables I and II correspond to the values collected and classified by increasing durations of the times of proper function and of the repair of the four centres, respectively.

The repair times are completed with a piece of information about the nature of the failure. The letters e, $\mathrm{m}, \mathrm{c}$ and o indicate the type of failure: electric, mechanic, computer or other, respectively.

\subsection{Exploitation of data}

The exploitation of the data requires the knowledge of the time distributions associated with the processes studied. The first step of this approach consists in testing the exponential distribution of the list of values. Non-parametric tests are used in order to determine the character of the distribution in comparison to the 


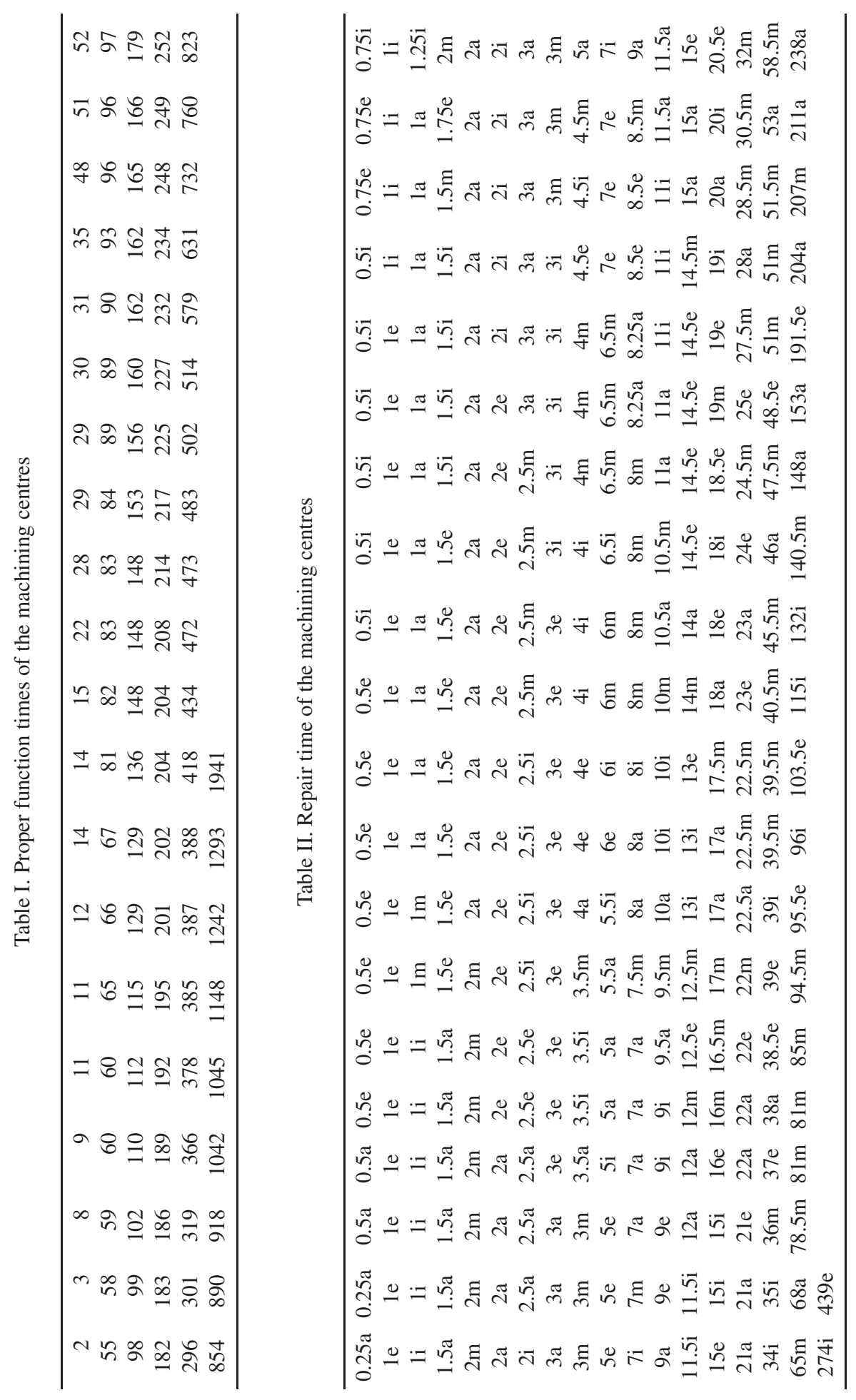




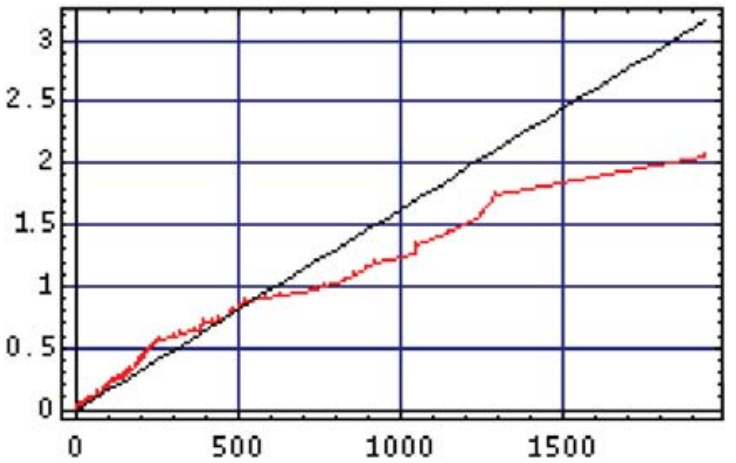

Figure 1. Proper function time distribution

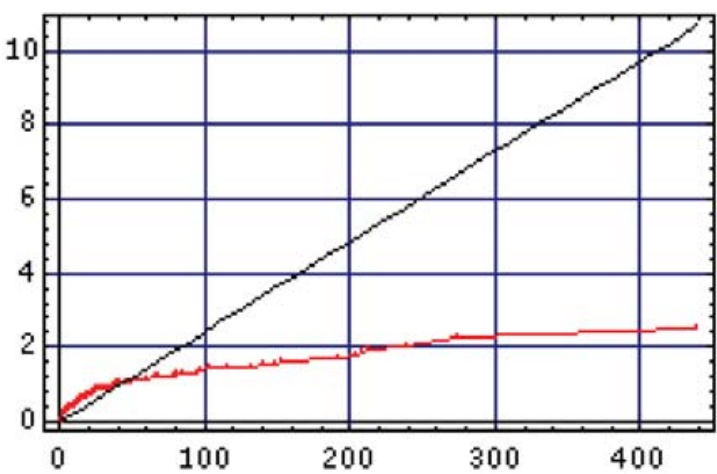

Figure 2. Time of repair distribution

exponential law. These tests guide one towards the choice of a law for the representation of the distribution of durations for the list of values. The conformity of the law in relation to the list of values can then be estimated by a classic test of goodness-of-fit.

\subsubsection{Non-parametric exponential tests}

Classically, the forms of distribution of factual data of production systems are compared to an exponential distribution. We proceed with a graphical analysis of the distribution of values of the sample according to two methods.

Test of linearity. In this test, the exponential law is represented by a straight line. The $n$ values of the sample are distributed along this line. The distance of the points from the line reveals the degree of fidelity of the distribution tested in relation to the exponential. The use of semi-logarithmic plotting paper is necessary for this test. The points, which correspond to $(n+1) /(n+1-i)$ as a function of each time $t_{i}(1 \leqslant i \leqslant n)$, are marked.

The analysis is visual. If the distance of the points is far from the straight line, the test does not permit the judgment of the degree of correlation between the two distributions. On the other hand, if the distribution of the points lies close to the straight line, the $\lambda$ parameter of the identified exponential law is given by

$$
\lambda=\frac{n}{\sum_{i=1}^{n} t_{i}}
$$

As far as the sample of reference is concerned, Figures 1 and 2 show the distribution of proper function and repair times. The distribution of the proper function time appears closer to the exponential (represented by the straight line) than the distribution of the time of repair which is remarkably further from the line.

Total time on test (TTT). This second test of exponentiality requires the analysis of the distribution of a transformed function defined for a sample of size $N$ by

$$
H_{F}^{-1}\left(\frac{i}{N}\right)=\sum_{j=1}^{i}\left(1-\frac{j-1}{N}\right)\left(X_{j}-X_{j-1}\right) \quad i=1, \ldots, N
$$

The mathematic expectation of the $F(t)$ distribution function is equal to $H_{F}^{-1}(1)$, which permits the introduction of the normalized TTT transformed function

$$
\left\|H_{F}^{-1}\left(\frac{i}{N}\right)\right\|=\frac{H_{F}^{-1}(i / N)}{H_{F}^{-1}(1)}
$$




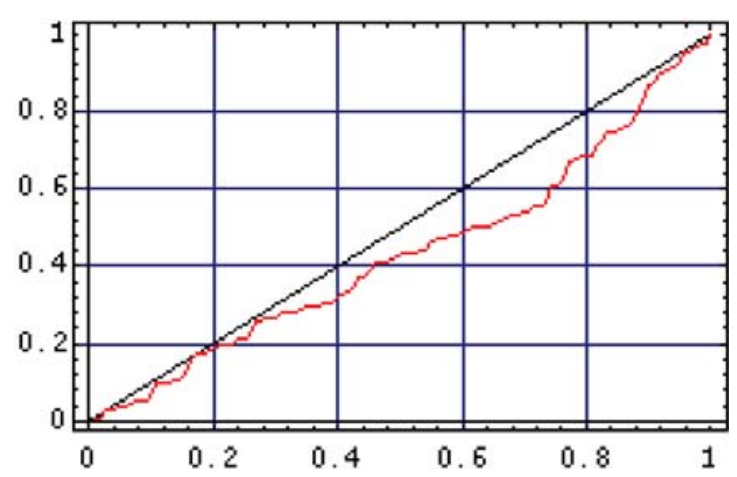

Figure 3. Proper function time distribution

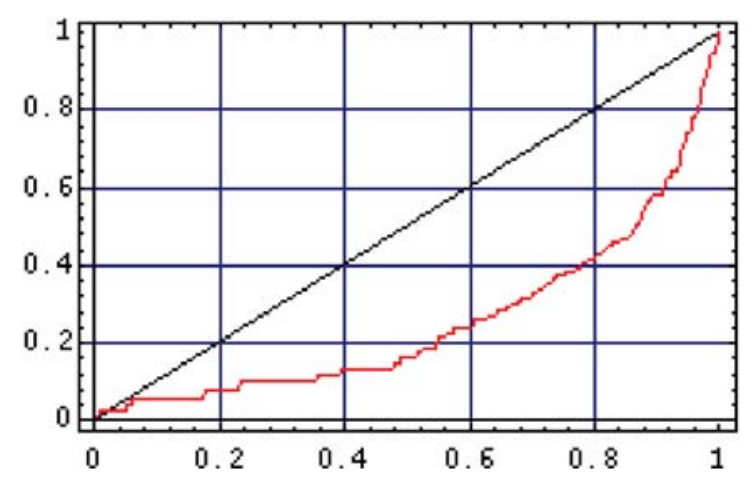

Figure 4. Time of repair distribution

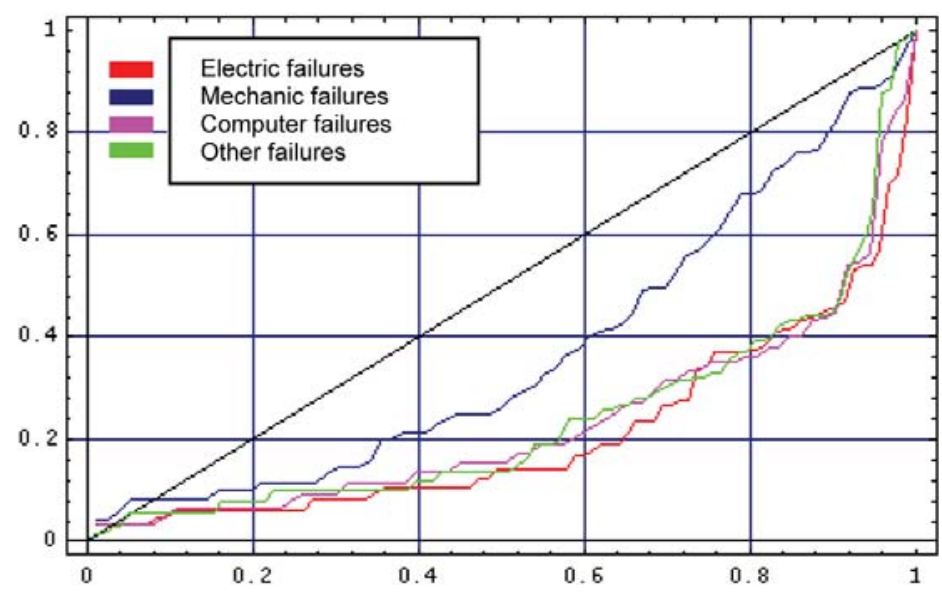

Figure 5. Repair times for each failure type

The normalized TTT transformed function has the following properties:

- the $X$ - and $Y$-axes of the representation are limited to one;

- the transformed function of the exponential distribution is represented by the first bisector (straight line at $45^{\circ}$ passing through the origin);

- the representations of the functions corresponding to increasing rates (hypo-exponential law) or decreasing rates (hyper-exponential law) are situated above and below the $45^{\circ}$ line, respectively.

The application of the exponential tests to the values of the tables (see Figures 3 and 4) show the following characteristics:

- the proper function times are approximately exponentially distributed (constant rates);

- the distribution of repair times follows a hyper-exponential law (decreasing hazard rate).

We can break down the sample of repair times according to the nature of the failures: electric, mechanic, computer and other. The analysis of the normalized TTT transformed function, associated with each of the distribution functions, shows (Figure 5) the same hyper-exponential character of repair times. 


\subsection{Characterization of the distributions}

As our objective is to identify the laws characterizing the distribution forms of failures and repairs, we limit our comments to continuous laws. Classically, five laws are considered for the identification of the processes taken into account in the evaluation models of dependability. These are:

- the exponential law, representing the distributions of proper function times during the period of maturity of equipment;

- the normal law, applied to the phenomena of wear or uncertainty;

- the log-normal law, often attached to the representation of the duration of repairs;

- the Weibull law, characterized by three parameters which make the law very flexible and favourable for the representation of the distribution of proper function times during the infant mortality and wear-out periods of the equipment; and

- the Erlang law, used for covering problems of material transport and durations of repair, flexible enough to allow the representation of numerous types of phenomena.

The characteristics of these laws (density function, distribution or survival functions, hazard rate, expectation, standard deviation, variance, etc.) are described in many works on statistics, so we will not describe them here. The identification of the parameters of the laws which approach the empirical distribution can be done analytically (calculations) or graphically (using probability plotting papers). The laws which represent the two lists of values are:

- for the proper function times, the exponential law with the parameter $\lambda=0.00375$;

- for the duration of repair, the normal law with parameters $\mu=7.85$ and $\sigma=42.49$, the log-normal law with parameters $\mu=1.66$ and $\sigma=1.47$ and the Weibull law with parameters $\beta=0.79$ and $\eta=10.88$.

\subsection{Goodness-of-fit tests}

Each method used to identify the laws delivers the parameters associated with the most closely related law. However, it does not mean that the identified law is the most representative of the distribution form of the list of values. Some goodness-of-fit tests exist. These non-parametric methods permit the attribution of a level of confidence between an adjusted law and the sample which it models. Two methods are classically encountered for the validation of the models: the chi-squared test and the Kolmogorov-Smirnov test.

The chi-squared test is applied to samples of a size greater than 50 values. It is based on the definition of classes and on the difference between the number of values contained in each theoretical and empirical class. The initial arranged sample is divided into $k$ intervals such that each one of them contains at least five values. The function of theoretical distribution is then split identically. The statistic of chi-squared based on the difference between the number of $F_{i}$ theoretical values and $f_{i}$ empirical values of each class $i$ is calculated by the expression

$$
E=\sum_{i=1}^{k} \frac{\left(f_{i}-F_{i}\right)^{2}}{F_{i}}
$$

$E$ is then compared to the table of chi-squared at $(k-1)-\rho$ degrees of freedom, $\rho$ corresponding to the number of estimated parameters of the law. If $E$ is greater than chi-squared, the theoretical law is not acceptable to the degree of corresponding confidence. This test tends to be precise enough in the case of homogenous laws. In the case of very dispersed laws, it is very sensitive to the distribution of values in the class. It is, then, preferable to use another test. The Kolmogorov-Smirnov test is less restrictive. The minimum size of the sample is not limited. The difference between the theoretical and empirical law is calculated point by point. The function of the empirical distribution is calculated by the method of median ranks according to the formula

$$
F^{*}\left(t_{i}\right)=\frac{\sum c_{i}}{N+1}
$$


where $\sum c_{i}$ is the number of failures accumulated at time $t_{i}$. The $F\left(t_{i}\right)$ theoretical values are calculated for each time $t_{i}$. The maximum absolute value of the difference between each empirical and theoretical value $(D N)$ is compared to the table value of $D N$, corresponding to the $\alpha$ risk. The theoretical hypothesis is rejected if

$$
D N=\operatorname{Max}\left\langle F^{*}\left(t_{i}\right)-F\left(t_{i}\right)\right\rangle>D N, \alpha
$$

The goodness-of-fit tests applied to the samples of reference allow for the acceptance of the exponential law of the parameter $\lambda=0.00375$ (distribution of proper functioning times) and the Weibull law of parameters $\beta=$ 0.79 and $\eta=10.88$ (distribution of repair times) with a level of confidence greater than $90 \%$. In contrast, the normal and log-normal laws tend not to be representative of the forms of distribution of the repair times contained in the sample of reference.

\section{DECOMPOSITION OF REPAIR TIMES}

The distribution forms of the samples representing the durations of failure or preventive interventions give useful information on the global behaviour of the equipment in relation to the maintenance strategies. However, the direct use of these results for the purpose of improvement is delicate. In fact, the collected values are an aggregation of different regrouped pieces of information. The data which could provide a distinction between the different stages of the process only express, in the majority of cases, the raw (i.e. not broken up into elementary times) value of down times.

For the repairs, this duration starts at the appearance of a failure and ends when the piece of equipment is again operational. The duration of preventive interventions begins with the time $t$ when the piece of equipment is available to the operators charged with its maintenance and ends when it reoccupies the position it had before the initialization of the intervention. However, between these two times several events take place. Each of these events represents a part of the global time of repair or preventive inspection and corresponds to a specific action. The ensemble of these actions constitutes the processes of corrective and preventive maintenance. It is then possible to decompose the global down time into elementary sequences corresponding to the phases of the maintenance processes. This decomposition simplifies the work of identifying the parameters at stake and to visualize the localization of their effects ${ }^{54}$. In this context, we propose a decomposition of the principal mechanisms of system maintenance under its corrective and preventive aspects.

\subsection{Corrective maintenance}

For the processes of repair, which take place between the time of failure and the moment when the piece of equipment is brought back into service, a partition into two phases can be established. The first step, leading to a diagnosis, corresponds to all the actions taken in order to identify the failure. The second step regroups all of the actions taken to repair the piece of equipment and put it back into service.

The decomposition may, however, be detailed at even further levels. We limit ourselves to the identification of phases at a generic level, to which are attributed the corresponding choices in the construction of a maintenance strategy. As such, the processes of repair are divided into six phases, presumed to be sequential and taking place within a specified duration. These six phases, illustrated in Figure 6, are as follows:

- $\varphi 1$ : detection and alert;

- $\varphi 2$ : awaiting intervention;

- $\varphi$ 3: diagnosis;

- $\varphi$ 4: awaiting repair;

- $\varphi 5$ : repair;

- $\varphi$ 6: return to service.

A phase of the decomposition may be of zero duration, since it may not exist in the process (a waiting time, for example) or it may exist in the form of a 'Dirac function' which appears as an event without any duration of time. 


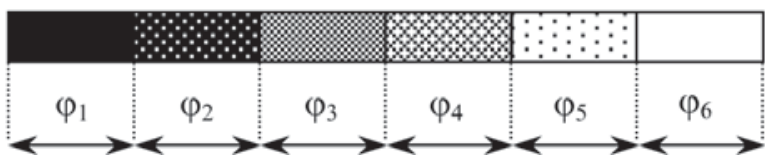

Figure 6. Corrective phase process

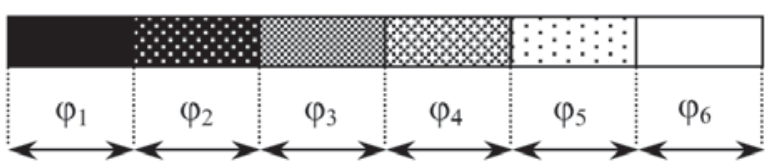

Figure 7. Phases of the preventive process

\subsection{Preventive maintenance}

The process of preventive maintenance, which begins at the moment the machine is made available for revision and ends with the return to its former functioning state, may be, in the same way, decomposed into phases. A segmentation of the process into two distinct phases makes two types of action appear when carrying out preventive maintenance. This classically begins by the implementation of a series of programmed operations. This first phase may be followed by an ensemble of operations, linked to the discovery of a certain number of defaults which require rapid intervention, without which the system would rapidly fail. Two types of response can be imagined. Either the system is returned to production and an agreement is made with the production party about a date when the system can be made available for adjustment or replacement of defective parts, or one may proceed directly to the repair of failed parts $\$$. The programmed phase is assigned a calculated amount of time. The following phase is of random duration, and in this phase repairs are made and decisions are taken in accordance with the production demands. As with the process of corrective maintenance, the decomposition of the duration of revision may be even more refined (see Figure 7). The phases are divided into segments as follows ${ }^{\S}$ :

- $\varphi 1$ : preparation of the unit;

- $\varphi$ 2: inspection and diagnosis;

- $\varphi$ 3: revision;

- $\varphi$ 4: awaiting repair;

- $\varphi$ 5: repair;

- $\varphi$ 6: return to service.

\section{INFLUENCE OF A MAINTENANCE STRATEGY ON ELEMENTARY TIMES}

In the following we apply ourselves to the decomposition of the processes and to the knowledge of the durations associated with each phase in order to study, by examination of the variations of these durations, the effect of the modifications given by the parameters of a maintenance strategy.

\footnotetext{
$¥$ A third possibility consists of allowing the system to function until its failure, but anticipating the problem by preparing the material and human resources required for repair. This is a 'programmed corrective action'.

$\S$ In the case of conditional maintenance, the appearance of precursor signs of failure generate a sequence of phases of the same nature as the corrective process.
} 


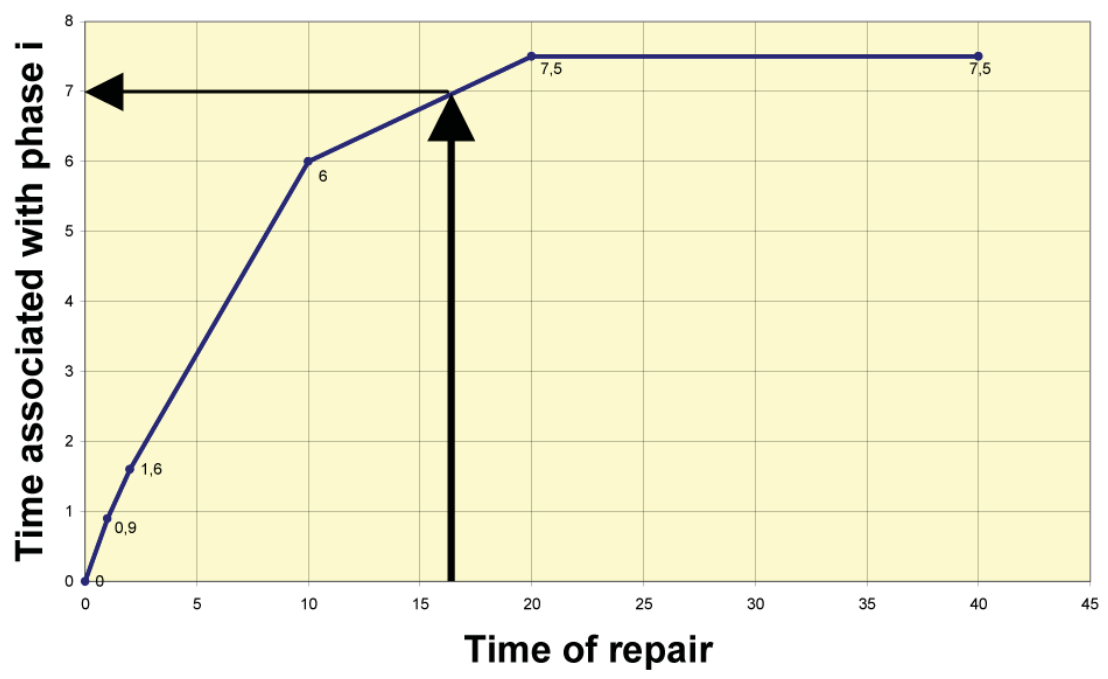

Figure 8. Duration of the phase $i$ as a function of the global duration

\subsection{Rules of decomposition}

It must be noted that the information on the duration of elementary phases are often not directly available. However, when the need is stated and the 'mechanisms' of capture exist, it is easy to establish the procedures which facilitate their capture. In the particular case where only raw data are accessible, corresponding to the global duration of the phenomenon (proper functioning time, failure time, revision time, etc.), the analysis will be global. Because we are in this case (i.e. we do not have the information about the times related to the elementary phases) we have opted for a reverse approach compared to what should normally be done in industry. Based on the previous results, the person in charge of maintenance would draw many lessons about the soundness of the maintenance parameters from the observation of the elementary phases. The lack of data incited us to do the opposite. We propose rules which, from the global duration of the operation (repair, revision, etc.) decomposed into an ensemble of $P$ phases, enables us to come up with the elementary duration corresponding to each phase. Each rule is an application

$$
R: D \stackrel{R}{\rightarrow} D_{i}
$$

with global duration of the operation $D, D_{i}$ as the elementary duration of the phase $i$ and

$$
D=\sum_{i=1}^{P} D_{i}
$$

The idea is not to define some standard distributions of each phase $D_{i}$ since they have been artificially built, but to give a form of representation when facing situations corresponding to some classical phenomena. In order to establish the existing relationship between $D$ and $D_{i}$, we rely on the knowledge of experts and on the knowhow and rules of the trade collected by consultations with maintenance departments. Two operations applied to $D$ appeared to be sufficient for the construction of the rules: multiplication by a lower-than-one coefficient and addition of a constant; representing, respectively, the proportion of the global duration and a fixed minimal duration. In Figure 8, a graphic illustration of the comprehension of the rules is proposed.

In this example, for an operation of 16 hours (the repair operation for example), 7 hours correspond to phase $i$ (phase of waiting for a part, for instance). 


\subsection{Application to the sample of reference}

We apply the set of steps leading to the decomposition of the processes on the initial sample list of values. Three types of failures are identified (electrical, mechanical and computer), a fourth class regroups the other types of failure.

\subsubsection{Decomposition of the repair processes}

In Table III we present the values given to the rules of decomposition. The duration $D_{i}$ associated with each phase $i$ can be estimated by calculating the proportion $p_{i}$ of the total value of the down time (represented by '*' in the table) and/or by addition of a fixed duration $f_{i}$ (represented by ' + ') $)^{\mathbb{I}}$. For example, for a repair time of 5 hours (belonging, then, to class 3 ) of electrical type, the duration $D_{3}$ of the phase $\varphi_{3}$ : diagnosis (bold numbers in Table III) is $D_{3}=5 \times 15 \%-0.1=0.65 \mathrm{~h}$.

We know that

$$
\Sigma D_{i}=D
$$

We can also write that

$$
D_{i}=p_{i}\left(D+f_{i}\right)
$$

with

$$
\Sigma p_{i}=1
$$

and

$$
\Sigma f_{i}=0
$$

From Equation (2) we can write that

$$
\Sigma D_{i}=\Sigma\left(p_{i}\left(D+f_{i}\right)\right)=D \Sigma p_{i}+\Sigma f_{i}
$$

Combined with Equations (3) and (4) this gives $\Sigma D_{i}=D$ which indeed corresponds to Equation (1).

We will limit our comments about Table III to the major considerations that helped us to establish the $p$ and $f$ coefficients as describing it in an exhaustive manner would take too long.

Each type of failure (electrical, mechanical and computer" ${ }^{\|}$) is itself divided into classes characterized by the duration of the failure. We distinguish five classes for each type of failure:

- immediate intervention carried out within the hour following the failure;

- rapid intervention needing, nonetheless, several actions to be taken;

- a long intervention likely to be caused by the complexity of the repair;

- delayed intervention which may be due to a prolonged waiting time for supplies;

- suspended intervention because of the unavailability of repair resources, for example.

According to the type of failure, the boundaries, or limits, of each class vary slightly. This is related to the fact that although the seriousness of the failure may be equal, mechanical interventions take longer than electrical or computer interventions. In fact, these interventions often require more manipulation, as the elements are

\footnotetext{
IThe precision of the numbers in Table IV may be surprising since they are based on the knowledge of experts, which is, of course, always subjective. In reality, these numbers were determined in order to express a continuity between the duration of phases in each of the classes of the table. We can see this continuity in Figure 8.

"For the 'other' types of failures, we consider that the rules of decomposition are the same as for the electrical repairs. Because of the extent of their possible durations and the diversity of their nature, electrical repairs are the least specific and those to which unknown failures may be most easily assimilated.
} 


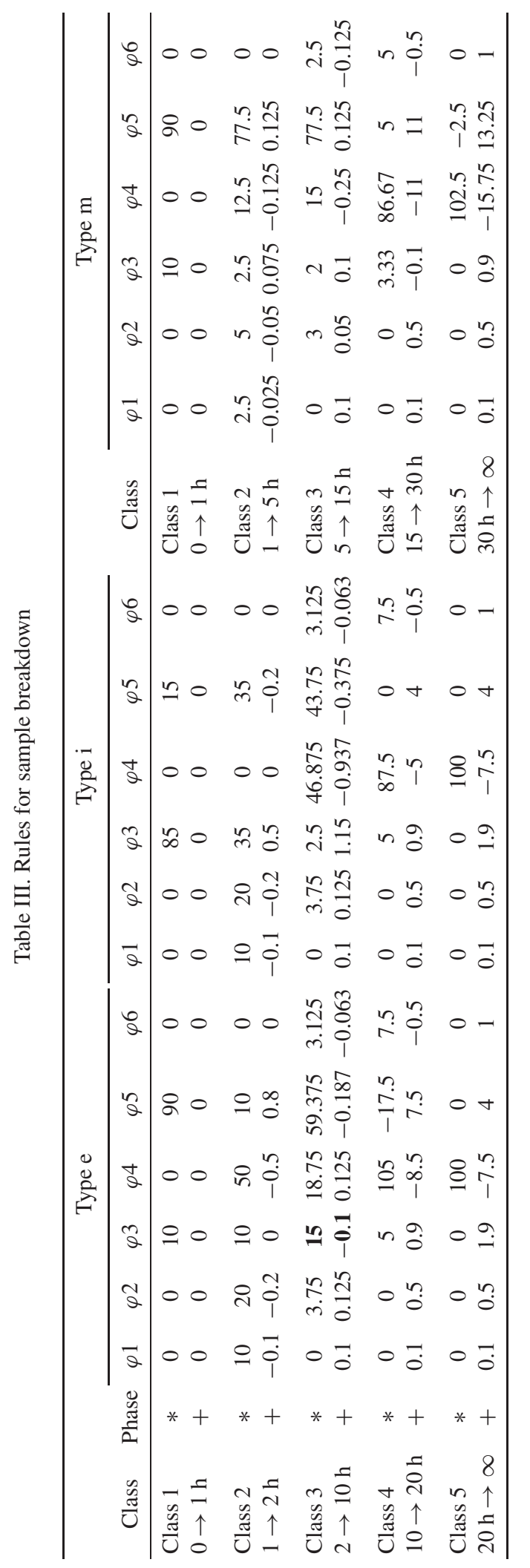




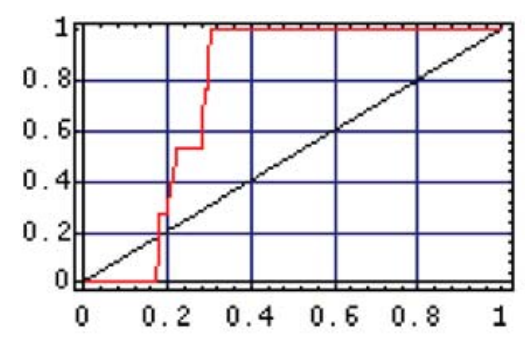

Phase 1: detection and alert

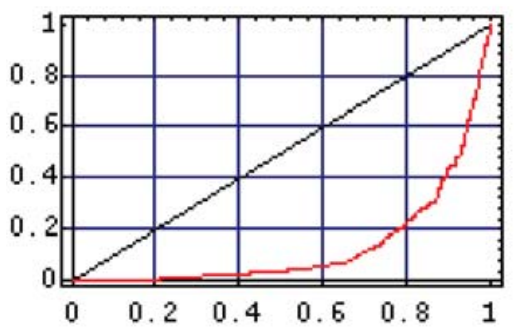

Phase 4: awaiting repair

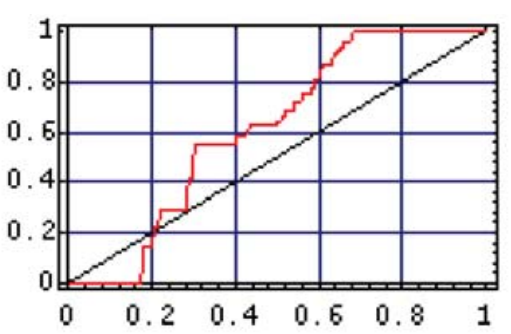

Phase 2: awaiting intervention

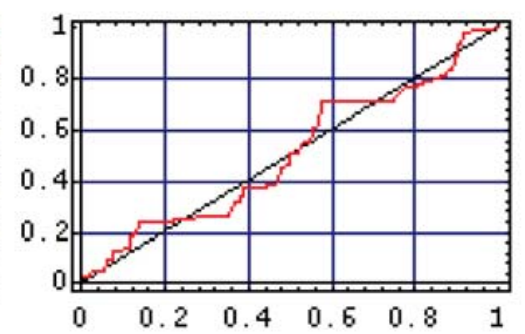

Phase 5: repair

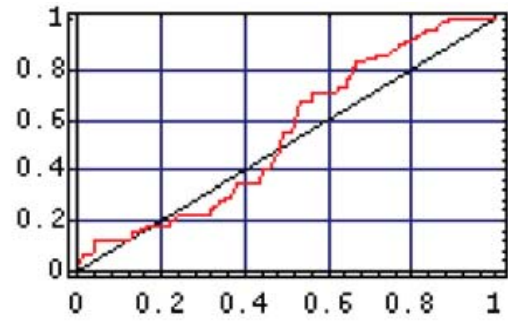

Phase 3: diagnosis

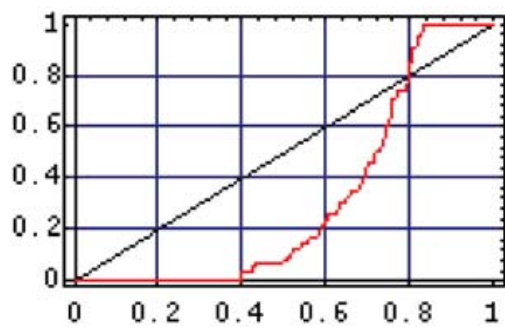

Phase 6: return to service

Figure 9. Distribution of the elementary phase times

frequently less accessible, heavier, more cumbersome, etc. On the other hand, the time needed for diagnosis is usually lower.

The $p$ and $f$ coefficients have been estimated by making the following assumptions.

- For very small $D(<1 \mathrm{~h})$, the main times correspond to the diagnosis and repair phases and are directly proportional to $D$.

- For medium $D(1 \mathrm{~h}<D<20$ or $30 \mathrm{~h})$, the total time is explained by the whole phase, which can be a percentage of $D$ but most often is a fixed coefficient representing an incompressible value added or subtracted from the proportional part.

- For large $D$ ( $>20$ or $30 \mathrm{~h}$ ), the immobilization is due to phase 4 , the waiting time corresponding to a spare parts delivery which has been delayed, for instance.

\subsubsection{Observation of the distributions}

After decomposing the repair times, it is interesting to consider the forms of distribution associated with the different phases. The hyper-exponential nature of the distribution of the sample of reference is not repeated for the whole of the distributions of the elementary phase times (Figure 9).

Certain distributions are, rather, hypo-exponential (detection and alert phases and awaiting diagnosis), others are more exponential (diagnosis, effective repair). The hyper-exponential curves are particularly marked at the level of the awaiting repair times which are most certainly at the origin of this singularity in the distribution of the global processes. The hyper-exponential distribution gives rise to a decreasing hazard rate. When applied to the processes of repair, this means that the more time goes by, the more the opportunity to repair diminishes. The very influential presence of the waiting and repair times (linked with the time for repair resources procurement) contributes to this decreasing character. Actually, the longer the duration of repair, the more there is a risk of a supply problem (implying a delayed repair).

Let us recall that the distribution functions result from the implementation of our own set of hypotheses. In a real industrial world, the approach would be reversed. From the distribution functions analysis, the engineer in charge of maintenance would determine if their forms correspond to a favourable or unfavourable situation and 


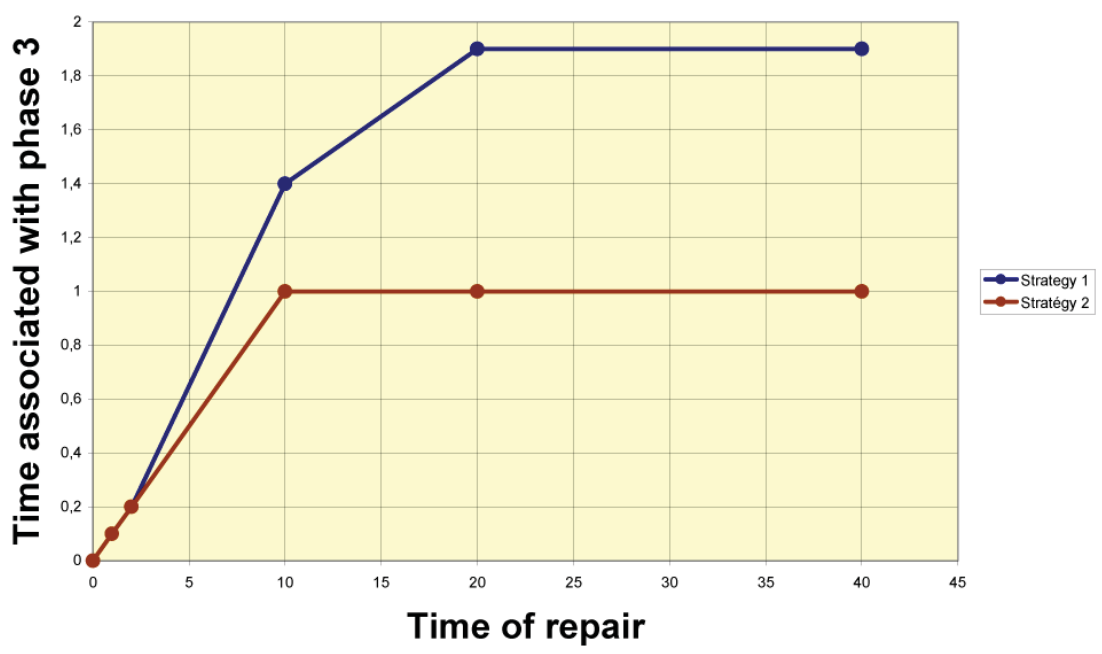

Figure 10. Duration of $\varphi_{3}$ according to the maintenance strategy

would explain the associated reasons. As the case may be, this analysis would allow one to reinforce or rethink the maintenance and logistic support strategies. In the situation that we have generated, the hyper-exponential form of the waiting times of repair would encourage one to modify the spare parts procurement strategy.

\subsection{Sensitivity of the system}

We propose the observation of the behaviour of the system when modified by the intermediary of a chosen variable, at the level of the distributions of the times of elementary phases. To this end, we establish a new set of rules. As opposed to the preceding table, these are not rules which are applied to the raw values of the times of repair. They concern the values obtained from the first set which are augmented or diminished according to the new maintenance variables. In other words, the new set of rules is applied to the $P$ samples (one per phase) generated by the first decomposition.

Again, let us take the example which served to illustrate the functioning of Table III. The previous value calculated for the phase of diagnosis corresponded to $D_{3}=0.65 \mathrm{~h}$. The value associated with this phase after having introduced the parameters representing the new strategy is now: $D_{3}^{\prime}=(0.65 \times 66.7 \%)+0.067=0.5 \mathrm{~h}$.

In Table IV we propose the rules resulting from the influence of the new set of variables.

The $P$ samples are modified in proportion to the influence of the new variables introduced in the maintenance strategy. The $P$ samples of the first decomposition are compared to those which are derived. This comparison between the forms of distributions may be carried out for each phase or globally.

As an illustration, we show in Figure 10 the variation of the length of the diagnosis phase as a function of the repair time for an electrical maintenance from the values in Tables III and IV. In order to establish the rules for Table IV, we consider that the given variables reflect an increase in the maintenance manpower (improvement of reactivity), a better stock of spare parts (reduction in supply time) and technologies that allow for better testing of the equipment (diminished diagnosis time).

The distributions of the rough and modified values of the complete process and of each of its phases can be compared. They are shown in Figures 11 and 12. The curves relating to the distributions of the modified sample are shown in black, the distributions of the elementary phases of the original sample are shown in grey.

The results show a high modification of the distributions of the complete process and of each of its phases. Generally speaking, these distributions evolve from the original hyper-exponential form (decreasing rate) toward an exponential (constant rate) and even hypo-exponential (increasing rate) tendency. The elementary times 1 , 2 and 6 (detection and alert, awaiting diagnosis and return to service), which do not occupy a very important place in the breakdown of the duration of repair, are only slightly modified. The pure repair (phase 5) remains 


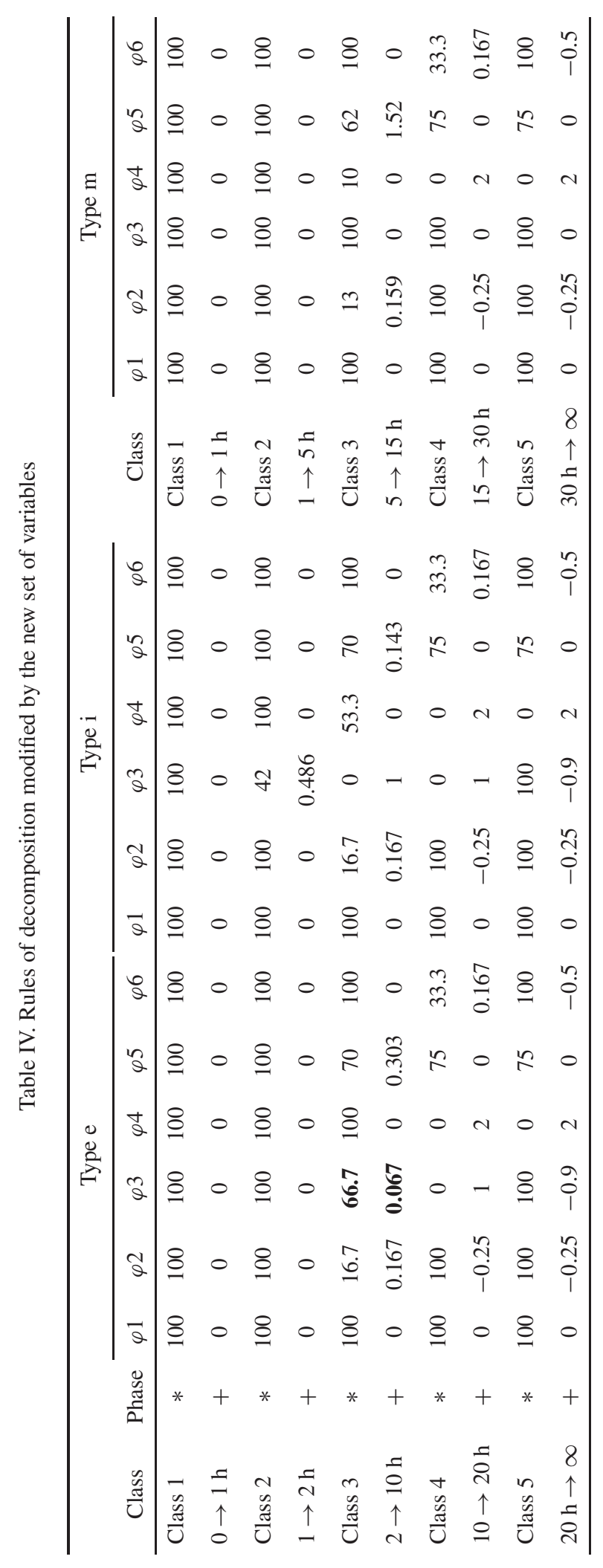




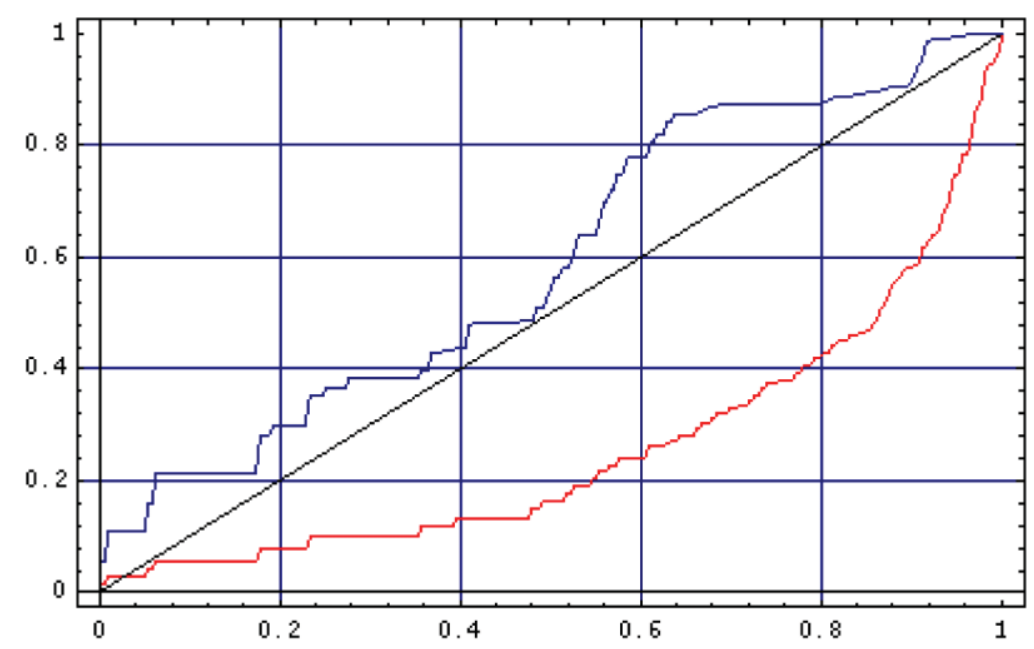

Figure 11. Distribution of the repair times

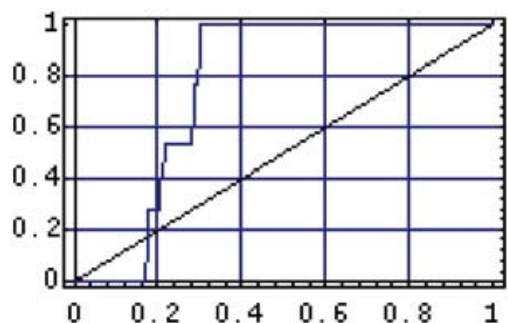

Phase 1: detection and alert

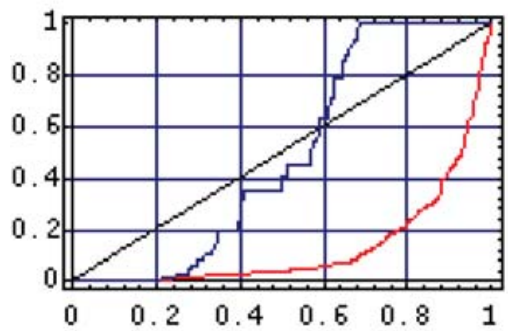

Phase 4: awaiting repair

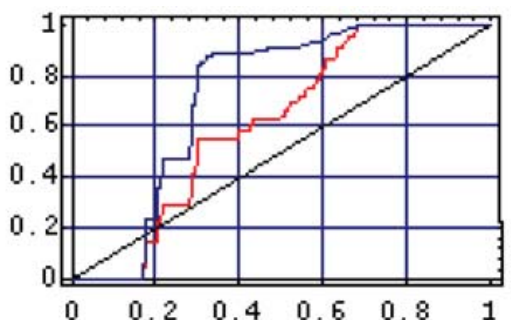

Phase 2: awaiting intervention

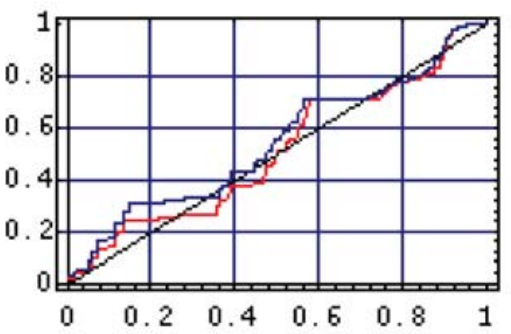

Phase 5: repair

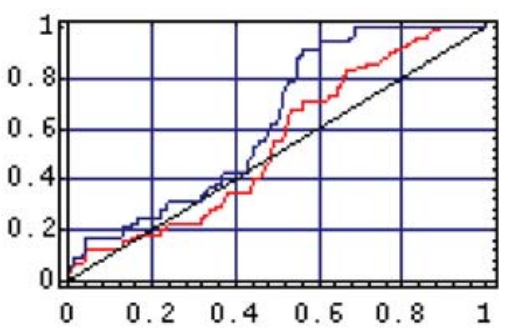

Phase 3: diagnosis

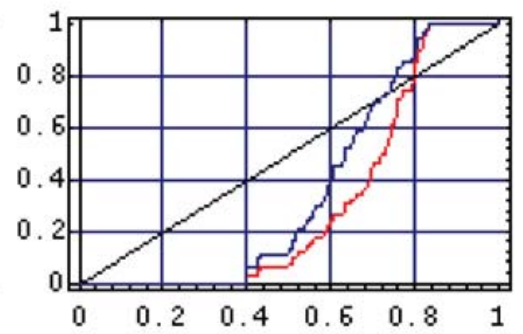

Phase 6: return to service

Figure 12. Distribution of the phases of the processes

sensitively distributed under exponential form after the new set of variables is applied. This statement allows one to assume that the non-exponential character, often stated in the analysis of repair time distributions, is often the result of parasite times like the waiting times, the pure repair rates remaining almost constant. Of course, these results must be qualified since they are set arbitrarily in this illustration. The values and explanations that we put forward in this example should not, then, always be taken as true. This is a principle that we wanted to illustrate. Let us be clear, also, that the observation of the forms of distribution is not a goal in itself. It is only the first step of a work of modelling the behaviour of the system to be evaluated from the angle of its form of reaction when a given strategy of maintenance is applied. 


\section{CONCLUSION}

After having made a bibliographic analysis, we have defined, in this work, a method for the evaluation of maintenance strategies coming from the analysis of factual data. The analysis of the state of the art was carried out by distinguishing between the works related to the maintenance strategies and those treating the forms of failure and repair. This analysis revealed a great diversity of works covering most of the characteristics of an industrial setting and strategies which are applied to it. We then recalled the different steps of processing the factual data. We have illustrated these steps with a typical case of a sample from an industrial setting, representative of a repair time of a tooling unit. When tests of exponentiality were applied, they showed broad ranges of repair times and particularities linked to the type of failure. The breakdown of the correction and prevention processes showed the presence of elementary phases that constitute the global down time. From the set-up of logical rules of construction, we have accessed certain pieces of information that were missing in order to assign to each phase its elementary duration. Because the effects of a strategy on the duration of the elementary phases were put forward, these enabled the building up of new samples that could be considered as performance indicators. By the measure of the impact on the global processes of the parameters having a local impact, this contribution should, then, offer industry a new means of evaluating the maintenance strategies that they implement.

\section{REFERENCES}

1. Kumar D. Proportional hazards modelling of repairable systems. Quality and Reliability Engineering International 1995; 11(5):361-369.

2. Cheng K, Zhang YL. Analysis for a consecutive- $k$-out-of- $n$ : repairable system with priority in repair. International Journal of Systems Science 2001; 32(5):591-598.

3. Lim TJ, Lie CH. Analysis of system reliability with dependent repair modes. IEEE Transactions on Reliability 2000; 49(2):153-162.

4. Pellegrin C. Choice of a periodic on-condition maintenance policy. International Journal of Production Research 1992; 30(5):1153-1173.

5. Lim TJ. Estimating system reliability with fully masked data under Brown-Proschan imperfect repair model. Reliability Engineering and System Safety 1998; 59(3):277-289.

6. Sim SH, Endreyeni J. A failure-repair model with minimal and major maintenance. IEEE Transactions on Reliability 1993; 42(1):134-140.

7. Paté-Cornell ME, Lee HL, Tagaras G. Warnings of malfunction: The decision to inspect and maintain production processes on schedule or on demand. Management Science 1987; 33(10):1277-1290.

8. Luce S. Choice criteria in conditional preventive maintenance. Mechanical Systems and Signal Processing 1999; 13(1):163-168.

9. Banerjee PK, Nichuiv N. Inspection policies for repairable systems. IIE Transactions 1996; 28(12):1003-1010.

10. Love CE, Guo R. Utilizing Weibull failure rates in repair limit analysis for equipment replacement preventive maintenance decisions. Journal of the Operational Research Society 1996; 47(11):1366-1376.

11. Tan JS, Kramer MA. A general framework for preventive maintenance optimization in chemical process operations. Computers and Chemical Engineering 1997; 21(12):1451-1469.

12. Logendran R, Talkington D. Analysis of cellular and functional manufacturing systems in the presence of machine breakdown. International Journal of Production Economics 1997; 53(3):239-256.

13. Kececioglu D, Sun FB. A general discrete-time dynamic programming model for the opportunistic replacement policy and its application to ball-bearing systems. Reliability Engineering and System Safety 1995; 47(3):175-185.

14. Vineyard ML, Meredith JR. Effect of a maintenance policies on FMS failures. International Journal of Production Research 1992; 2647-2657.

15. Hopp WJ, Sung-Chi W. Machine maintenance with multiple maintenance actions. IIE Transactions 1990; 22(3): 226-233.

16. Gupta YP, Chand S. Strategies of replacement under Markov renewal process. International Journal of Information and Management Sciences 1993; 4(1):41-50. 
17. Bee DD, Pickrell BD, Bea RG, Roberts KR, Williamson RB. Safety Management Assessment System (SMAS): A process for identifying and evaluating human and organization factors in marine system operations with field test results. Reliability Engineering and System Safety 1999; 65(2):125-140.

18. Sridharan V, Mohanavadivu P. Some statistical characteristics of a repairable, standby, human and machine system. IEEE Transactions on Reliability 1998; 47(4):431-435.

19. Johnson C. Representing the impact of time on human error and systems failure. Interacting with Computers 1998; 11(1):53-86.

20. Moss B. Failure by design. New Scientist 1999; 162(2184):59.

21. Haken B. Design: The path to success or failure?. Electronic Engineering 1998; 70(858):77.

22. Papazoglou IA. Bayesian decision analysis and reliability certification. Reliability Engineering and System Safety 1999; 66(2):177-198.

23. Archibald TW, Dekker R. Modified block-replacement for multiple-component systems. IEEE Transactions on Reliability 1996; 45(1):75-83.

24. Levitin G, Lisnianski A, Ben-Haim H, Elmakis D. Redundancy optimization for series-parallel multi state systems. IEEE Transactions on Reliability 1998; 47(2):165-172.

25. Ohnishi M, Morioka T, Ibaraki T. Optimal minimal-repair and replacement problem of discrete-time Markovian deterioration system under incomplete state information. Computers and Industrial Engineering 1994; 27(1-4): 409-412.

26. Hontelez JAM, Burger HH, Wijnmalen DJD. Optimum condition-based maintenance policies for deteriorating systems with partial information. Reliability Engineering and System Safety 1996; 51(3):267-274.

27. Makis V. Optimal control policy for a tool-wear process subject to shocks and random failures. International Journal of Production Economics 1999; 60(1):613-621.

28. Kapur PK, Bhalla VK. Optimum maintenance policies maximizing service reliability. International Journal of Systems Science 1990; 21(1):217-224.

29. Haugen K, Hokstad P, Sandtorv H. The analysis of failure data in the presence of critical and degraded failures. Reliability Engineering and System Safety 1997; 58(2):97-107.

30. Hopp WJ, Kuo YL. An optimal structured policy for maintenance of partially observable aircraft engine components. Naval Research Logistics 1998; 45(4):335-352.

31. Ciampoli M. A probabilistic methodology to assess the reliability of deteriorating structural elements. Computer Methods in Applied Mechanics and Engineering 1999; 168(1-4):207-220.

32. Vanderperre E, Makhanov SS, Suchatvejapoom S. Long-run availability of a repairable parallel system. Microelectronics and Reliability 1997; 37(3):525-527.

33. Dagpunar JS. A maintenance model with opportunities and interrupt replacement options. Journal of the Operational Research Society 1996; 47(11):1406-1409.

34. Srinivasan MM, Lee HS. Production-inventory systems with preventive maintenance. IIE Transactions 1996; 28(11):879-890.

35. Dedopoulos IT, Smeers Y. An age reduction approach for finite horizon optimization of preventive maintenance for single units subject to random failures. Computers and Industrial Engineering 1998; 34(3):643-654.

36. Kvam PH, Singh H. Estimating reliability of components with increasing failure rate using series system data. Naval Research Logistics 1998; 45(1):115-123.

37. Vaurio JK. On time-dependent availability and maintenance optimization of standby units under various maintenance policies. Reliability Engineering and System Safety 1997; 56(1):79-89.

38. Tseng ST. Optimal preventive maintenance policy for deteriorating production systems. IIE Transactions 1996; 28(8):687-694.

39. Hassett TF, Dietrich DL, Szidarovszky F. Time-varying failure rates in the availability and reliability analysis of repairable systems. IEEE Transactions on Reliability 1995; 44(1):155-160.

40. Siddiqui SA, Mishra S. Reliability analysis of a finite range failure model. Microelectronics and Reliability 1995; 35(5):859-862.

41. Christer AH. Developments in delay time analysis for modelling plant maintenance. Journal of the Operational Research Society 1999; 50(11):1120-1137.

42. Sheu SR, Jhang JP. Optimal age and block replacement policies for a multi-component system with a shock type failure interaction. International Journal of Systems Science 1998; 29(8):805-817.

43. Liu HM. Reliability of a load-sharing k-out-of-n:G system: Non-iid components with arbitrary distributions. IEEE Transactions on Reliability 1998; 47(3):279-284.

44. Gopalan N, Kumar UD. Probabilistic analysis of a n-unit cold-standby system with general failure and repair time distributions. Microelectronics and Reliability 1995; 35(5):851-857. 
45. Albin SL, Seng C. Preventive replacement in systems with dependent components. IEEE Transactions on Reliability 1992; 41(2):230-238.

46. Coetzee IL. The role of nhpp models in the practical analysis of maintenance failure data. Reliability Engineering and System Safety 1997; 56(2):161-168.

47. Hagin AA. Reliability evaluation of a repairable network with limited capacity and structural redundancy. Microelectronics and Reliability 1997; 37(2):341-347.

48. Sherwin DJ. Inspect or monitor? Engineering Cost and Production Economics 1990; 18(3):223-231.

49. Barbera F, Schneider H, Kelle P. A condition-based maintenance model with exponential failures and fixed inspection intervals. Journal of the Operational Research Society 1996; 47(8):1037-1045.

50. Salameh MK, Jaber MY. Optimal lot sizing with regular maintenance interruptions. Applied Mathematical Modelling 1997; 21(2):85-90.

51. Waganer LM. Comparing maintenance approaches for tokamak fusion power plants. Fusion Technology 2001; 39(2):458-461.

52. Wang KH, Lee HC. Cost-analysis of the cold-standby M/M/R machine repair problem with multiple modes of failure. Microelectronics and Reliability 1998; 38(3):435-441.

53. Iravani SMR, Duenyas I, Olsen TL. A production/inventory system subject to failure with limited repair capacity. Operations Research 2000; 48(6):951-964.

54. Pérès F. Outils d'analyse de performance pour stratégies de maintenance dans les systèmes de production. PhD Thesis, Université Bordeaux I, 1996.

\section{Authors' biographies}

François Pérès is a Production Engineer who graduated from the Ecole Nationale d'Ingénieurs de Tarbes (France). Following his PhD in Maintenance Management from Bordeaux I University, he is now at Ecole Centrale Paris (ECP) where he works as an Assistant Professor. Head of the production and maintenance courses, he has been involved in many industrial projects with companies working in various fields like aeronautics, nuclear, transport, etc. Belonging to the 'Laboratoire Génie Industriel' of ECP, he is responsible for an area of research called 'Conception et Exploitation de Systèmes Sûrs'; its main research concerns the aspects of dependability, maintenance and logistic support.

Daniel Noyes is a Professor at the Ecole Nationale d'Ingénieurs de Tarbes (France), Director of the 'Laboratoire Génie de Production' of ENIT and is responsible of the 'Production Automatisée' research team of the LGP. The activities of the research team deal with dependability of production systems, management of abnormal operating modes, data flow architectures and some automation problems of production systems. His current research interests concern dependability evaluation and performance analysis of production systems with the use of stochastic process theory tools such as Markov models, as well as concurrent engineering in the product development process. 